\title{
Sharpening of enhanced backscattering peak in a disordered gain medium
}

\author{
Shechao Charles Feng \\ Department of Physics, University of California, Los Angeles, California 90095-1547 \\ and Department of Physics, Hong Kong University of Science and Technology, Clear Water Bay, Kowloon, Hong Kong \\ Zhao-Quing Zhang \\ Department of Physics, Hong Kong, University of Science and Technology, Clear Water Bay, Kowloon, Hong Kong
}

(Received 22 May 1995; revised manuscript received 22 August 1995)

\begin{abstract}
We study theoretically the phenomenon of enhanced backscattering (EBS) from a bulk disordered gain medium due to the presence of a dye and a pump beam. We show that the presence of the gain, in combination with the effect of saturation, can dramatically sharpen the EBS peak function. In the particular case of a point source incidence for the probe beam along with the presence of a plane-wave pump beam, the EBS approaches a $\delta$ function in angular space.
\end{abstract}

The phenomenon of enhanced backscattering (EBS) of light from a bulk disordered medium constitutes perhaps the clearest example of the importance of phase coherence in optical propagation in the multiple-scattering regime. Following its first experimental observation in the early 1980s, ${ }^{1}$ this effect has received much attention in the optics community. ${ }^{2,3}$ The scientific importance of the EBS is further enhanced by its direct analogy to the quantum correction of conductance in disordered metals at low temperatures due to weak localization, ${ }^{4}$ as both effects originate from the perfect phase coherence of time-reversed interfering multiplescattering paths in the backscattering direction. More recently, the phenomenon of EBS has been analyzed in the context of light scattering from random rough surfaces, 5,6 and it is hoped that the EBS peak function can be used to extract useful information about the geometrical and scattering properties of the random surfaces under study.

The study of EBS has been mainly confined to various random systems in linear optics. Recently, several authors started to address the issue of what would happen in the nonlinear optical regime. In particular, Kravtsov, Agranovich, and Grigorishin ${ }^{7}$ showed that in the case of secondharmonic generation (due to $\chi_{2}$ nonlinear susceptibility ${ }^{8}$ ) the EBS peak height for second-harmonic light is very much reduced from the usual factor of 2 , due to the restriction that only second-harmonic light generated near the sample surface contributes to the EBS signal, whereas those generated anywhere in the bulk contribute to the diffuse background. Later Agranovich and Kravtsov ${ }^{9}$ predicted that when the $\chi_{3}$ nonlinear susceptibility ${ }^{8}$ is taken into account, the EBS peak function of linear optics must be modified by the appearance of a narrow "dip" very close to the backscattering direction, although this result has recently been challenged by Heiderich, Maynard, and van Tiggelen. ${ }^{10}$

In this paper, we study how the EBS peak is modified in a disordered multiple-scattering medium by a different source of nonlinearity, namely when the probe light can gain intensity due to stimulated emission. Initial treatment of this problem was provided by Zyuzin recently, ${ }^{11}$ who, however, did not take into account the important effect of saturation. We shall devise a simple model in the present paper to address this physical effect.
A concrete, experimentally relevant, system we have in mind is a dye-dissolved fluid containing a high concentration of scatterers (e.g., polystyrene spheres). For simplicity, we model the dye as a three-level atomic system with energies $E_{1}<E_{2}<E_{3}$ such that level $E_{2}$ is metastable. If a pump beam at frequency $\omega_{p}=\left(E_{3}-E_{1}\right) / \hbar$ is applied to the system, population inversions between $E_{2}$ and $E_{1}$ can be achieved, such that a probe light at frequency $\omega=\left(E_{2}-E_{1}\right) / \hbar$ passing through the sample will experience gain. Let us denote the gain coefficient as $\mu_{g}$ (in units of $\mathrm{cm}^{-1}$ ). Another important feature of a disordered gain medium is the nonlinear effect of saturation. If the gain coefficient were to remain a constant, it is evident from physical ground that for a sufficiently large system, the probe light intensity will become exponentially amplified to infinity. This scenario is not permitted on physical ground, because when the probe light intensity increases, the population inversion decreases, which causes $\mu_{g}$ to decrease, such that in steady state, a saturation value for the probe light intensity is reached, which is a finite fraction of that of the pump light. Our main conclusion in this paper is that the presence of positive gain and the constraint of saturation in combination can induce a dramatic sharpening of the EBS peak, up to the limit of a delta function when the incident probe beam is of the form of a point source.

The geometry that we consider is the standard semiinfinite space where $z>0$ defines the region of the multiplescattering medium. We assume the incident pump beam is approximately a continuous (cw) plane wave, with a width $W$ large compared to all the other length scales in the problem. The incoherent diffuse optical intensity of the pump beam can be obtained from the diffusion equation

$$
-D_{p} \frac{d^{2}}{d z^{2}} \phi_{p}(z)=I_{p}\left[\delta\left(z-l_{p}^{*}\right)-\delta\left(z-l_{p}^{*}+a_{p}\right)\right],
$$

where $\phi_{p}(z)$ is a function of $z$ alone due to the assumption of a plane-wave incidence (in the normal direction), $l_{p}^{*}$ is the transport mean free path of the pump beam, $D_{p}=c l_{p}^{*} / 3$ the corresponding diffusion coefficient, $I_{p}$ is the flux of the incident pump beam, and $a_{p}=2\left(l_{p}^{*}+z_{0 p}\right)$ is the distance between the diffusive source and its image. We have followed 
here the standard treatment of the boundary condition for the diffusion approximation for incoherent light transport in a multiple-scattering medium, and assumed $\phi_{p}=0$ at the extrapolated boundary $z=-z_{0}$ with $z_{0}$ being a parameter dependent on the contrast in the index of retraction at the interface (e.g., $z_{0 p} \approx 0.7 l_{p}^{*}$ for the index-matched case). Solving Eq. (1) yields the result that the pump light intensity inside the medium for $z>l_{p}^{*}$ is approximately a constant, given by

$$
\phi_{p}(z)=\frac{a_{p} I_{p}}{2 D_{p}}, \text { valid for } z>l_{p}^{*}
$$

Now we consider the incoherent light intensity for the probe beam at $\omega=\left(E_{2}-E_{1}\right) / \hbar$. Let us first discuss the case where the incident probe beam is also a cw plane wave, with optical flux $I$, and beam width $W \gg l^{*}, l_{p}^{*}$, where $l^{*}$ is the transport mean free path for the probe beam. For simplicity, we approximate the gain process at $\omega$ phenomenologically by a constant gain coefficient $\mu_{g}$, together with a cutoff-type saturation procedure, as we now illustrate.

If the incident source were not a cw but an impulse, i.e., $S(z, t)=I\left[\delta\left(z-l^{*}\right)-\delta\left(z-l^{*}+a\right)\right] \delta(t) \quad$ where $a=2\left(l^{*}\right.$ $\left.+z_{0}\right)$, then the light intensity at time $t$ should be described by the time-dependent diffusion equation

$$
\frac{\partial}{\partial t} \phi(z, t)-D \frac{\partial^{2}}{\partial z^{2}} \phi(z, t)-c \mu_{g} \phi(z, t)=S(z, t),
$$

where $D=c l^{*} / 3$. The solution is simply given by the timedomain one-dimensional (1D) diffusion propagator

$$
\begin{aligned}
\phi(z, t)= & \frac{I}{\sqrt{4 \pi D t}}\left\{\exp \left[-\frac{\left(z-l^{*}\right)^{2}}{4 D t}\right]\right. \\
& \left.-\exp \left[-\frac{\left(z-l^{*}+a\right)^{2}}{4 D t}\right]\right\} e^{c \mu_{g} t} .
\end{aligned}
$$

We see that with a constant gain coefficient, the light intensity response to an impulse at $t=0$ for the probe beam diverges as $t \rightarrow \infty$. This is clearly unphysical; so we impose a saturation condition by introducing a phenomenological saturation time parameter $T$, such that the intensity response to a plane wave cw source $S(z, t)=I\left[\delta\left(z-l^{*}\right)-\delta(z\right.$ $\left.\left.-l^{*}+a\right)\right]$ is given by

$$
\begin{aligned}
\phi(z)= & I \int_{0}^{\infty} \frac{d t}{\sqrt{4 \pi D t}}\left\{\exp \left[-\frac{\left(z-l^{*}\right)^{2}}{4 D t}\right]\right. \\
& \left.-\exp \left[-\frac{\left(z-l^{*}+a\right)^{2}}{4 D t}\right]\right\} e^{f(t)},
\end{aligned}
$$

where $f(t)=c \mu_{g} t$ for $t \leqslant T$ and $f(t)=c \mu_{g} T$ for $t>T$. It is clear that $T$ models physically, albeit crudely, the nonlinear saturation effect which cuts off gain when the probe light intensity reaches a certain threshold value, corresponding to a finite fraction of the pump light density in the medium.

Equation (5) can be rewritten in the form

$$
\phi(z)=I e^{c \mu_{g} T}\left[\int_{0}^{\infty} P_{0}(z, t) d t-\int_{0}^{T} P_{0}(z, t) g(t) d t\right],
$$

where $g(t)=1-e^{-c \mu_{g}(T-t)}$, and $P_{0}(z, t)$ is the $1 \mathrm{D}$ diffusion propagator in the absence of gain:

$$
\begin{aligned}
P_{0}(z, t) \equiv & \frac{1}{\sqrt{4 \pi D t}}\left[\exp \left(-\frac{\left(z-l^{*}\right)^{2}}{4 D t}\right)\right. \\
& \left.-\exp \left(-\frac{\left(z-l^{*}+a\right)^{2}}{4 D t}\right)\right] .
\end{aligned}
$$

We shall see later that the condition $c \mu_{g} T \gg 1$ usually holds. In this case, for $t$ within the range of integration $0<t<T$ in the second term of Eq. (6), the function $g(t)$ can be well approximated by $g(t) \approx \theta\left(T^{\prime}-t\right) \quad$ (step function) with $T^{\prime}=T-1 / c \mu_{g} \approx T$, since $g(t=T)=0$ exactly. Thus we arrive at the approximate expression

$$
\phi(z) \approx I e^{c \mu_{g} T} \int_{T^{\prime}}^{\infty} P_{0}(z, t) d t .
$$

After some simple manipulations, we arrive at a form for $\phi(z)$ :

$$
\phi(z) \approx \frac{a I}{2 D} e^{c \mu_{g} T} E\left(\frac{z+z_{0}}{\sqrt{4 D T^{\prime}}}\right),
$$

where $E(x) \equiv 2 / \pi \int_{0}^{x} e^{-y^{2}} d y$ is an error function, and $z_{0} \approx 0.7 l$ for index-matched interface. The behavior of $\phi(z)$ is quite simple, it rises linearly for $z \ll z_{T}$, and saturates at $\phi_{\infty}=(a I / 2 D) e^{c \mu_{g} T}$ for $z \gg z_{T}$, where $z_{T} \equiv \sqrt{4 D T^{\prime}}$ is a diffusive depth associated with the saturation time $T$.

The saturation time parameter can be determined experimentally by making the physical saturation requirement $\phi\left(z \gg z_{T}\right)=\gamma \phi_{p}$ where the constant percentage factor $\gamma<1$ is a property of the dye material. Thus we can write

$$
c \mu_{g} T=\ln \frac{\gamma I_{p}}{I}+\ln \frac{a_{p} D}{a D_{p}} \approx \ln \frac{\gamma I_{p}}{I},
$$

i.e., $T$ is mainly determined by the ratio of incident pump light intensity and that of the probe light. So if $I$ is chosen to be much smaller than $\gamma I_{p}$, the condition $T \gg 1 / c \mu_{g}$ is easily fulfilled.

In order to calculate the EBS peak function in the presence of gain, we need to calculate the following $\mathrm{cw}$ diffusion propagator $[\vec{r} \equiv(\vec{\rho}, z)]$

$$
\begin{aligned}
Q(\vec{\rho}, z) & =\int_{0}^{T} e^{c \mu_{g} t} P_{0}(\vec{\rho}, z ; t) d t+\int_{T}^{\infty} e^{c \mu_{g} T} P_{0}(\vec{\rho}, z ; t) d t \\
& \approx e^{c \mu_{g} T} \int_{T^{\prime}}^{\infty} P_{0}(\vec{\rho}, z ; t)
\end{aligned}
$$

where $P_{0}(\vec{\rho}, z ; t)$ is the $3 \mathrm{D}$ time-dependent diffusion propagator without gain, given by

$$
\begin{aligned}
P_{0}(\vec{\rho}, z ; t) \equiv & \frac{1}{(4 \pi D t)^{3 / 2}}\left[\exp \left(-\frac{\rho^{2}+\left(z-l^{*}\right)^{2}}{4 D t}\right)\right. \\
& \left.-\exp \left(-\frac{\rho^{2}+\left(z-l^{*}+a\right)^{2}}{4 D t}\right)\right] .
\end{aligned}
$$

Setting $y^{2}=1 / 4 D T, Q(\vec{\rho}, z)$ can be rewritten as 


$$
\begin{aligned}
Q(\vec{\rho}, z)= & \frac{e^{c \mu_{g} T}}{4 \pi D} \frac{2}{\sqrt{\pi}} \int_{0}^{1 / z_{T}} d y\left[e^{-y^{2}\left[\rho^{2}+\left(z-l^{*}\right)^{2}\right]}\right. \\
& \left.-e^{-y^{2}\left[\rho^{2}+\left(z-l^{*}+a\right)^{2}\right]}\right]
\end{aligned}
$$

where $z_{T}=\sqrt{2 D\left(T-1 / \mu_{g}\right)} \approx \sqrt{2 D T}$. In the linear optics case without gain, we let $T \rightarrow 0$ and $z_{T} \rightarrow 0$ in the above expression, and we recover the familiar expression ${ }^{2}$

$$
\begin{aligned}
Q(\vec{\rho}, z)= & \frac{1}{4 \pi D\left[\rho^{2}+(z-l)^{2}\right]^{1 / 2}} \\
& -\frac{1}{4 \pi D\left[\rho^{2}+(z-l+a)^{2}\right]^{1 / 2}} .
\end{aligned}
$$

The $z$-dependent incoherent light intensity $\phi(z)$ is related to the propagator $Q(\vec{r})$ simply by $\phi(z)=\int d^{2} \rho Q(\vec{r})$. Following Akkermans, Wolf, and Maynard, ${ }^{2}$ the albedo function $\alpha\left(\hat{k}_{i}, \hat{k}_{f}\right)$ (defined as the ratio of detected optical flux per unit area per solid angle in the detection direction $\hat{k}_{f}$ to the incident flux of the probe light) contains a contribution from the incoherent re-radiated light as well as that due to the coherent interference between the time-reversed multiplescattering paths, which has the form

$$
\alpha\left(\hat{k}_{i}, \hat{k}_{f}\right)=\frac{c}{4 \pi} \int d^{2} \rho[1+\cos (\vec{q} \cdot \vec{\rho})] Q\left(\vec{\rho}, z \approx l^{*}\right),
$$

where $\vec{q}$ is the projection of $\vec{k}_{i}+\vec{k}_{f}$ in the $x-y$ plane, which measures the angular deviation from the exact backscattering direction where the phase coherence of the time-reversed multiple-scattering paths is perfect. Near normal incidence, $q \equiv|\vec{q}| \approx k \theta$ where $\theta$ is the detector angle measured from the backscattering direction. After some algebra, we find

$$
\alpha(\theta)=\frac{c e^{c \mu_{g} T}}{(4 \pi)^{2} D} \frac{2}{\sqrt{\pi}}[I(0,0)-I(0, a)+I(q, 0)-I(q, a)],
$$

where

$$
I(q, z)=\int d^{2} \rho \cos (\vec{q} \cdot \vec{\rho}) \int_{0}^{1 / z_{T}} d y \exp \left[-y^{2}\left(\rho^{2}+z^{2}\right)\right] .
$$

In the absence of gain, it has been shown ${ }^{2}$ that

$$
\alpha(\theta) \approx \alpha_{\mathrm{inc}}\left(1+\frac{1-e^{-q a}}{q a}\right),
$$

where $\alpha_{\text {inc }}$ is the incoherent background. This can be obtained from our formalism by taking the limit $T \rightarrow 0$. Thus the angular width of the EBS peak in linear optics is $\Delta \theta \approx 1 / k a$ and the height of the EBS peak relative to the incoherent background is a factor of 2 . In the presence of gain, it follows from Eq. (16) by setting $q=0(\theta=0)$ that the relative EBS height is still a factor of 2 .

For $q \neq 0$, using the following integral involving the Bessel function $J_{0},{ }^{12}$

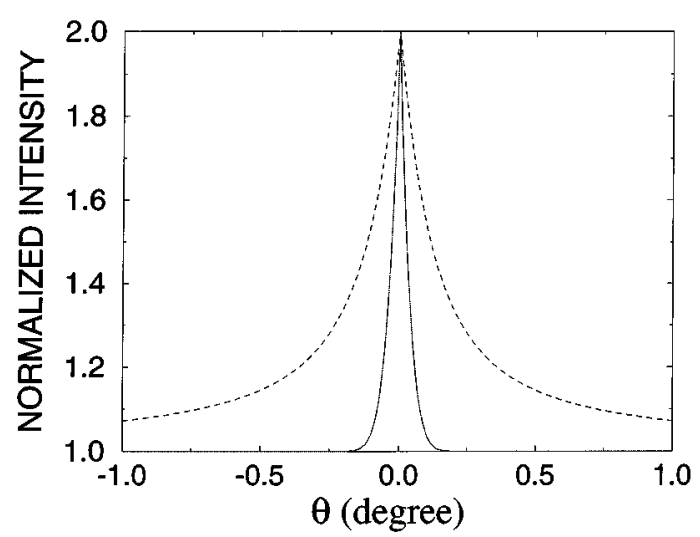

FIG. 1. Comparison of normalized EBS peak function $\alpha(\theta) / \alpha_{\text {inc }}$ in the absence (dotted line) to that in the presence of gain (solid line). We choose a saturation time parameter $T \approx 15 \mathrm{ps}$. Other parameters chosen are $c \approx 3 \times 10^{8} \mathrm{~m} / \mathrm{s}, \quad l^{*}=19 \mu \mathrm{m}$, $\lambda=0.515 \mu \mathrm{m}$, corresponding to realistic experimental values. This gives a saturation depth $z_{T}=107 \mu \mathrm{m} \gg l^{*}$.

$$
\int_{0}^{\infty} x e^{-\alpha x^{2}} J_{0}(\beta x) d x=\frac{1}{2 \alpha} e^{-\beta^{2} / 4 \alpha} .
$$

$I(q, z)$ may be rewritten as

$$
I(q, z)=\pi \int_{0}^{1 / z_{T}} d y \frac{\exp \left(-z^{2} y^{2}-q^{2} / 4 y^{2}\right)}{y^{2}} .
$$

For $q \neq 0$, setting $x=q / y, I(q, z)$ may be written in the following form:

$$
I(q, z)=\frac{\pi}{q} \int_{q z_{T}}^{\infty} e^{f(x)}
$$

where

$$
f(x)=-\frac{(q z)^{2}}{x^{2}}-\frac{x^{2}}{4} .
$$

For $q z_{T} \gg 1, I(q, z)$ can be approximately evaluated using the method of steepest descent, with the result

$$
I(q, z) \approx \frac{\pi \sqrt{\pi}}{2 q} e^{-q z} e^{-\left(q z_{T}\right)^{2}}
$$

leading to the following expression for the albedo function:

$$
\alpha(\theta) \approx \alpha_{\text {inc }}\left[1+\frac{1}{2}\left(\frac{1-e^{-q a}}{q a}\right) e^{-\left(q z_{T}\right)^{2}}\right]
$$

which is valid for $\theta \gg 1 / k z_{T}$. Since $z_{T} \gg a$, the angular width of the EBS in the presence of gain is reduced to $\Delta \theta \approx 1 / k z_{T}$. As $z_{T}$ depends on the ratio of the incident pump beam intensity and that of the probe beam, it should be interesting to observe experimentally how the EBS beam width gets reduced when the pump beam intensity is increased while keeping that of the probe beam fixed.

In Fig. 1, we illustrate the sharpening effect due to gain by plotting the normalized albedo $\left[\alpha(\theta) / \alpha_{\text {inc }}\right]$ in the absence (dotted line) as well as in the presence of gain (solid line). We choose a saturation time parameter $T \approx 15 \mathrm{ps}$, and $c \approx 3 \times 10^{8} \mathrm{~m} / \mathrm{s}, l^{*}=19 \mu \mathrm{m}, \lambda=0.515 \mu \mathrm{m}$, corresponding to 
realistic experimental values. This gives a saturation depth $z_{T}=107 \mu \mathrm{m} \gg l^{*}$. Thus the angular width in the absence of gain (dotted line) is $\Delta \theta \approx \pi / k a \approx 0.22^{\circ}$ and the angular width in the presence of gain (solid line) is $\Delta \theta \approx \pi / k z_{T} \approx 0.03^{\circ}$. The effect of the narrowing and sharpening of the EBS peak is quite obvious.

We also remark that in the usual linear optics regime without gain, the diffusion approximation usually used is not really valid for $\Delta \theta \geqslant 1 / \mathrm{ka}$ as at these large angles only scattering paths of order a few mean free paths contribute to $\alpha(\theta)$. But in the presence of gain, the albedo is dominated by long scattering paths, thus the diffusion approach becomes a very good approximation for the problem.

The above discussion is based on the assumption of a plane-wave probe beam incidence. In the case where the incident probe beam is focused to an area $A$ of order $l^{2}$ (since $l \gg \lambda \equiv 2 \pi / k$, we can still use the notion of a well-defined incident wave vector $\vec{k}_{i}$ ), the sharpening effect of the EBS peak due to gain is even more dramatic. In this case, the diffusive probe-light intensity is simply

$$
\phi(\vec{\rho}, z)=I A Q(\vec{\rho}, z),
$$

assuming the incident light is focused at the origin $\vec{\rho}=0$. Since in the saturation regime, we must have

$$
\phi(\vec{\rho}, z)=\gamma \phi_{p}(z)
$$

and since $\phi_{p}$ is $\rho$ independent, we must require a $\rho$-dependent saturation time $T(\rho)$ such that $\phi$ is independent of $\rho$. From Eqs. (22), we see that this implies a $\rho$-independent 3D propagator $Q\left(\rho, z \approx l^{*}\right)$ ! Using Eqs. (15), (2), (22), and (23), we find that in this case the EBS peak function is sharply peaked, i.e., we may write

$$
\alpha(\theta)=\frac{c W^{2} \gamma I_{p} a}{8 \pi D_{p} I A}\left(1+e^{-k^{2} W^{2} \theta^{2}}\right),
$$

where $W \gg l^{*}$ is simply the width of the pump beam which can be made arbitrarily large! This effect of extreme sharpening of the EBS peak in the presence of gain with a point source incidence probe beam deserves immediate experimental verification.

S.C.F. wishes to thank A. Lagendijk especially for hospitality during his three month visit in 1991 at the University of Amsterdam, and early discussions during this period on this and related subjects which motivated him to study this problem. S.C.F. also thanks the Hong Kong University of Science and Technology for his three month visit in 1994 during which the main part of the present work was conducted. We also thank K.-M. Yoo for many fruitful discussions. This work was supported in part (at UCLA) by the ONR under Grant No. N00014-92-J-4004 (the Medical Free Electron Laser Program), the DOE under Grant No. DEFG03-88ER45378, and the NIM Inc. of Philadelphia.
${ }^{1}$ M. P. van Albada and L. Lagendijk, Phys. Rev. Lett. 55, 2692 (1985); P. E. Wolf and G. Maret, ibid. 55, 2696 (1985).

${ }^{2}$ E. Akkermans, P. E. Wolf, and R. Maynard, Phys. Rev. Lett. 56, 1471 (1986); P. E. Wolf, G. Maret, E. Akkermans, and R. Maynard, J. Phys. (France) 49, 63 (1988).

${ }^{3}$ Y. Kuga and A. Ishimaru, J. Opt. Soc. Am. A 1, 831 (1984); M. Kaveh, M. Rosenbluh, I. Edrei, and I. Freund, Phys. Rev. Lett. 57, 2049 (1986).

${ }^{4}$ G. Bergmann, Phys. Rep. 107, 1 (1984); P. A. Lee and T. V. Ramakrishnan, Rev. Mod. Phys. 57, 287 (1985).

${ }^{5}$ A. A. Maradudin, E. R. Mendez, and T. Michel, in Scattering in Volumes and Surfaces, edited by M. Nieto-Vesperinas and J. C. Dainty (North-Holland, Amsterdam, 1990); A. A. Maradudin et al., Waves Random Media 1, S129 (1991).

${ }^{6}$ E. Jakeman, IEE Colloquium on Radar Clutter and Multipath Propagation, Digest No. 62, 66 (1989); F. M. Ismagilov and Y. A. Kravtsov, Waves Random Media 2, 203 (1992); M. -J. Kim, J. C. Dainty, A. T. Friberg, and A. J. Sant, J. Opt. Soc. Am. A 7, 569 (1990).
${ }^{7}$ V. E. Kravtsov, V. M. Agranovich, and K. I. Grigorishin, Phys. Rev. B 44, 4931 (1991).

${ }^{8} \chi_{2}$ and $\chi_{3}$ are defined by the following expansion of the relation between the displacement vector $\vec{D}$ and the electrical field $\vec{E}$ :

$$
\vec{D}=\vec{E}+4 \pi \chi_{1} \vec{E}+\chi_{2} \cdot \vec{E} \vec{E}+\chi_{3} \cdot \vec{E} \vec{E} \vec{E},
$$

where $\chi_{1}$ is the usual linear optical susceptibility with $\epsilon=1+4 \pi \chi_{1} . \quad \chi_{2}$ is a second-rank nonlinear optical tensor which is responsible for the well-known phenomenon of secondharmonic generation. Similarly, $\chi_{3}$ is a third-rank next-order nonlinear optical tensor.

${ }^{9}$ V. M. Agranovich and V. E. Kravtsov, Phys. Rev. B 43, 13691 (1991).

${ }^{10}$ A. Heiderich, R. Maynard, and B. A. van Tiggelen, Opt. Commun. 15, 392 (1995).

${ }^{11}$ A. Yu. Zyuzin, Europhys. Lett. 26, 517 (1994).

${ }^{12}$ I. S. Gradshteyn and I. M. Ryzhik, Table of Integrals, Series, and Products (Academic, New York, 1980). 